\title{
The Introduction of a Prescribing Ward Round to Reduce Prescribing Errors on a Pediatric Intensive Care Unit
}

Walsh A, Booth R, Rajani K, Cochrane L, Peters MJ, du Pré P

\begin{abstract}
Our paediatric intensive care unit (PICU) performs active surveillance for prescribing errors and detects a mean of 1.66 with an SD of 0.18 total prescription errors per occupied bed day. The primary aim of this project was to reduce the number of prescribing errors in PICU. The secondary aims were to improve the workflow in the unit and reduce the time staff spent on medication queries/prescribing. We introduced a daily multidisciplinary prescribing round to our PICU. Prescribing errors reduced, with the mean number of total prescription errors per bed day falling from $1.66(0.18)$ to $1.19(0.13)$, the mean number of clinical prescription errors per bed day falling from $0.46(0.09)$ to $0.3(0.07)$, and the mean number of non-clinical prescribing errors per bed day falling from $1.12(0.15)$ to $0.67(0.1)$. Forty-eight staff responded to the survey, 39 of whom had been directly involved in the rounds. The majority ( 37 of $39 ; 95 \%$ ) said the prescribing round reduced the overall time they spent on prescribing/medication queries during their shift, and 9 of 10 (90\%) prescribers said that they were interrupted fewer times for medication queries while doing other tasks. Almost all (47 of $48 ; 98 \%)$ said that they thought the prescribing ward round should continue. Introduction of a prescribing round with senior medical and pharmacist involvement was associated with a reduction in prescribing errors as well as reduction in the overall time staff spent on medication queries and prescribing. The round was well received by staff, with $98 \%$ wanting it to continue.
\end{abstract}

\section{Summary:}

A prescribing ward round was introduced on a paediatric intensive care unit (PICU).

\section{The Problem:}

Medication errors are a common cause of harm to patients, with approximately 237 million medication errors occurring in England every year. An estimated 66.1million of these errors are potentially clinically significant.1 Medication errors are also a significant financial burden to the National Health Service, costing an estimated $f 750$ million each year. 2 Prescribing errors form a large proportion of medication errors. The complexity of paediatric prescribing means that children are more likely than adults to suffer harm as a result of a medication error.3 $\mathrm{A}$ systematic review of hospital medication administration errors in children found little evidence for the effectiveness of a single intervention in reducing the error rate. 4 A recent survey of paediatric hospitals in England showed that the electronic prescribing systems in use do not prevent the majority of harmful error scenarios from being prescribed. 5 While electronic prescribing may remove some non-clinical errors such as legibility and failure to use 
international non-proprietary names, it does not prevent incorrect drugs, doses or rates from being prescribed and may introduce new errors such as duplication. One major contributing factor may be the practice of electronic prescribing during major distractions such as ward rounds or at busy central desks with multiple interruptions from telephones and staff. Our PICU performs active surveillance for prescribing errors, and at the time this project started surveillance detected a mean error rate of 1.66 with an SD of 0.18 total prescription errors per occupied bed day. The mean number of clinical prescription errors (with potential to affect the patient) per bed day was 0.46 (0.09), and the mean number of nonclinical prescribing errors per bed day was 1.12 (0.15) (table 1). The most common errors were duplication, medicines not being discontinued, incorrect doses, rates and frequencies, and incorrect prescribing of drug levels. The majority of the errors did not result in harm to patients.

\section{Aims:}

The primary aim was to reduce the number of prescribing errors in PICU. The secondary aims were to improve the workflow in the unit and reduce the time staff spent on medication queries/prescribing. We planned to review the impact of the round after 2 months.

\section{Making a Case for Change:}

Our high detection of prescribing errors was on the departmental risk register. These were discussed at our weekly morbidity and mortality and monthly risk action group meetings. Following discussion with pharmacy, medical and nursing staff, a daily multidisciplinary prescribing round was planned to help address this problem. We decided on a start date for the prescribing round. Our plan was shared at unit meetings and by emails and posters.

\section{Your Improvements:}

We introduced a prescribing round to PICU in December 2018. This took place every weekday at approximately 11:00. It involved a senior fellow and clinical pharmacist visiting each bed space and addressing any prescription queries and prescribing non-urgent medicines. It was designed to improve multidisciplinary team collaboration and ensure that potential errors were identified at the time of prescribing and that any drug or dose queries were addressed in real time. The prescribing error data continued to be collected daily by the pharmacy team and analysed by the Quality and Safety team. A staff survey was circulated 2 months after the intervention was introduced to gain feedback on the round. After the intervention was introduced, prescribing errors reduced, with the mean number of total prescription errors per bed day falling from 1.66 $(0.18)$ to $1.19(0.13)$ (figure 1 ), the mean number of clinical prescription errors per bed day falling from $0.46(0.09)$ to $0.3(0.07)$ (figure 2$)$, and the mean number of non-clinical prescribing errors per bed day falling from $1.12(0.15)$ to 0.67 (0.1) (figure 3). Forty-eight staff responded to the survey, 39 of whom had been directly involved in the rounds. The majority ( 37 of $39 ; 95 \%$ ) said the prescribing round reduced the overall time they spent on prescribing/medication 
queries during their shift, and 9 of 10 prescribers (90\%) said that they were interrupted fewer times for medication queries while doing other tasks. Almost all $(47$ of $48 ; 98 \%)$ said that they thought the prescribing ward round should continue. One person (2\%) was concerned that the senior fellow on the floor was unavailable for other tasks during the round. Following this feedback, we ensured the round was conducted as efficiently as possible and that other staff were available to manage other patient queries. Staff were keen for the round to happen 7days a week and as close to the designated time as possible. All 48 said that they thought the prescribing ward round improved medication safety and reduced errors which are reflected in our prescribing error data. We disseminated the results of the survey in our monthly departmental newsletter.

\section{Learning and Next Steps:}

Introduction of a prescribing round with senior medical and pharmacist involvement was associated with a reduction in prescribing errors as well as reduction in the overall time staff spent on medication queries and prescribing. The round was well received by staff, with $98 \%$ wanting it to continue. We feel that the introduction of the prescribing round reduced the prescribing error rate because close involvement of the pharmacist at the prescribing stage meant that they were frequently able to identify and address prescribing issues that were not highlighted by our electronic prescribing system, thereby preventing many errors that would have otherwise slipped through. Involving the entire multidisciplinary team in the design and establishment of the project was key to its success. Staff availability, in particular pharmacist availability, was the biggest obstacle to establishing the round. Undoubtedly this adds an additional ward round to the day that already includes an antimicrobial stewardship round; however, the benefit gained by reducing the number of interruptions to the rest of the day was felt to outweigh this concern. It is imperative that the round is run as efficiently as possible to minimise 'ward round fatigue'. Timing of the round is also crucial, as pharmacists need to have enough time to have clinically reviewed the charts beforehand, but the round needs to happen early enough in the day that prescribing is not unnecessarily delayed. Our Trust introduced a new electronic patient prescribing system 4months after the introduction of the prescribing round, at which time the prescribing round had to be suspended due to time constraints. During the time that the prescribing round was suspended, the clinical prescribing error rate increased, with the mean number of clinical prescription errors per bed day rising to $0.67(0.08)$, which may have been associated with the introduction of the new electronic prescribing system. We have recently resumed the prescribing round and plan to expand to other intensive care units in our Trust.

\section{References:}

1. Elliott RA, Camacho E, Campbell $F$ et al. Prevalence and economic burden of medication errors in the NHS in England. Policy Research Unit in Economic Evaluation of Health and Care Interventions. 22 February 2018. 
2. National Patient Safety Agency. Safety in doses: medication safety incidents in the NHS. 2007.

3. Kaushal R, Bates DW, Landrigan $C$ et al. Medication errors and adverse drug events in pediatric inpatients. JAMA. 2001;285(16):2114-2120.

4. Ameer A, Dhillon S, Peters MJ, Ghaleb M. Systematic literature review of hospital medication administration errors in children. Integrated Pharmacy Research and Practice. 2015;4:153-165.

5. Fox A, Portlock J, Brown D. Electronic prescribing in paediatric secondary care: are harmful errors prevented? Archives of Disease in Childhood. 2019;104:895-899.

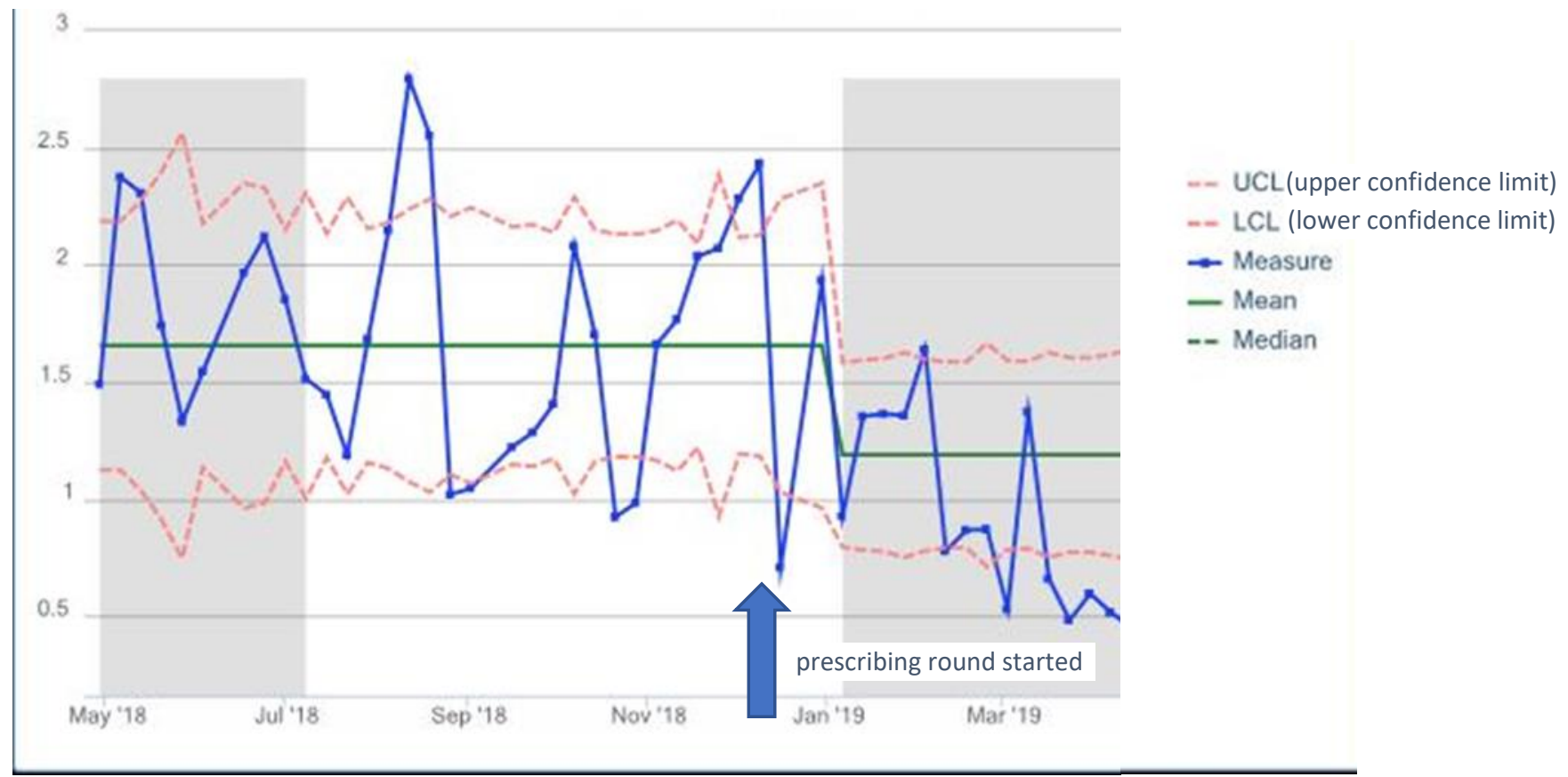

Figure 1. PICU total prescription errors per bed day 


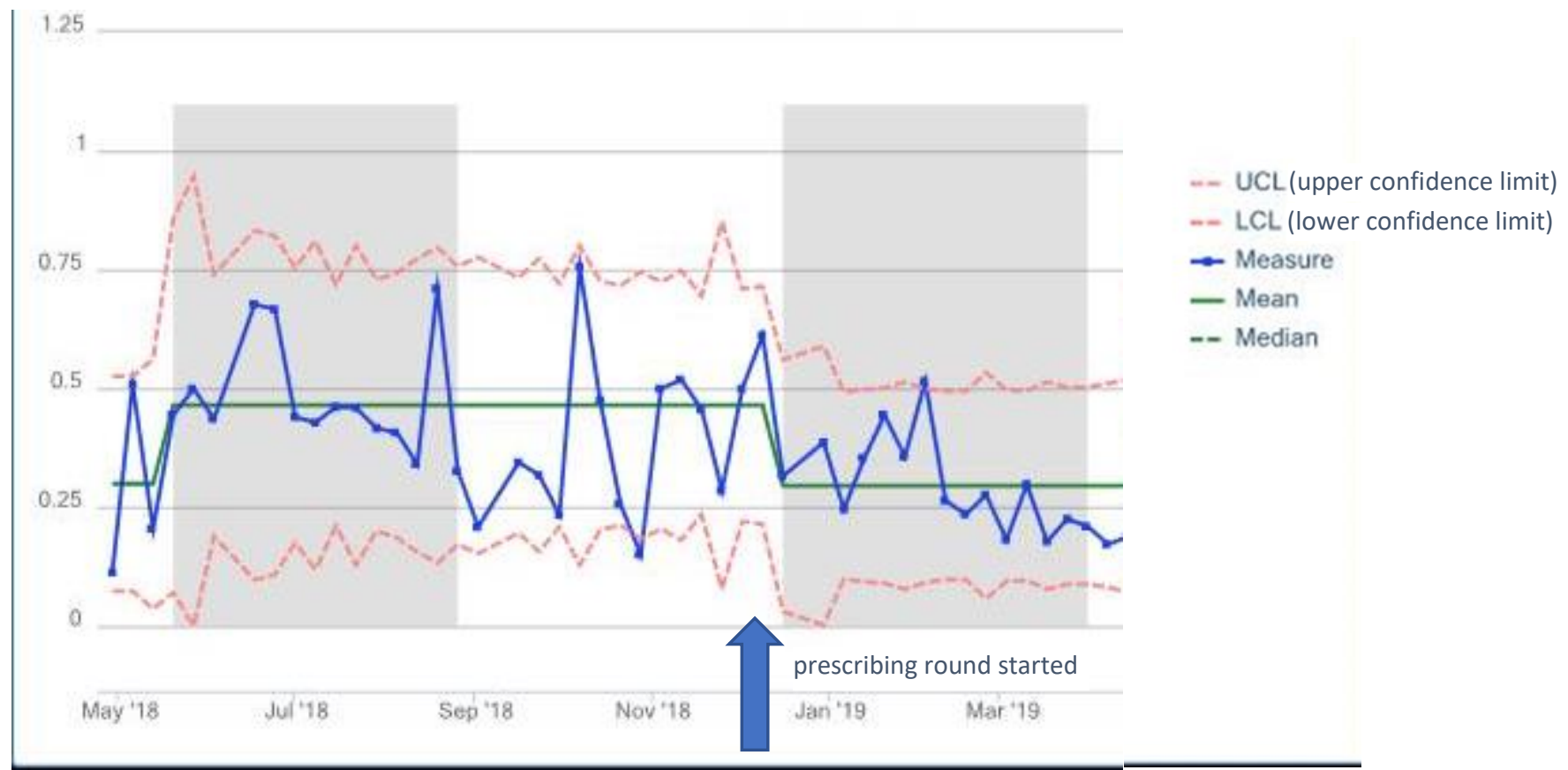

Figure 2. PICU clinical prescribing errors per bed day

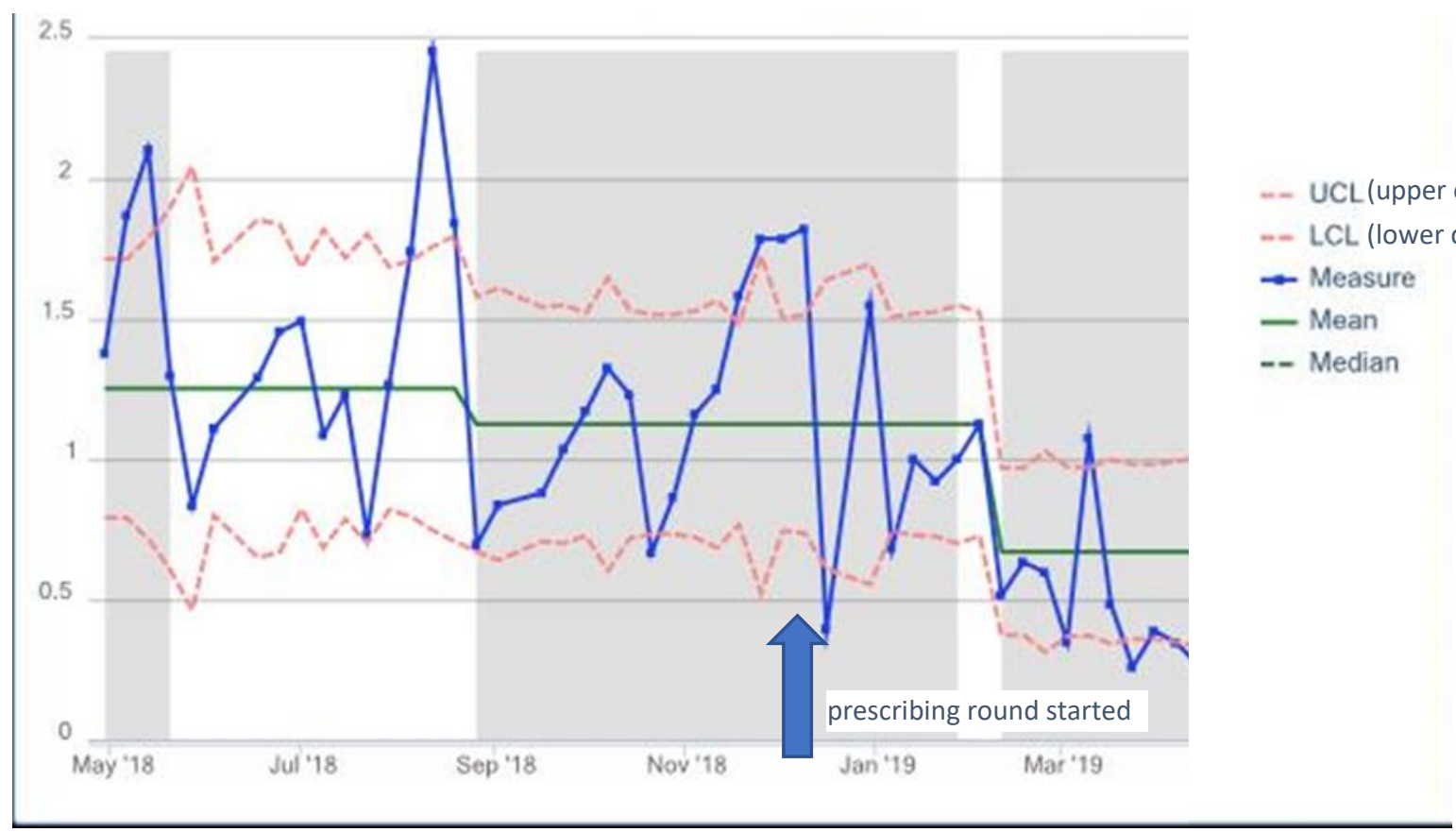

Figure 3. PICU non-clinical prescribing errors per bed day 


\begin{tabular}{|l|l|}
\hline Clinical prescribing errors & Non-clinical prescribing errors \\
\hline $\begin{array}{l}\text { Incorrect/missing patient details (allergy status, } \\
\text { weight) }\end{array}$ & Prescription not discontinued \\
\hline $\begin{array}{l}\text { Incorrect/missing drug, dose, units, strength, } \\
\text { frequency, route, concentration, rate or diluent }\end{array}$ & Duplicate \\
\hline Omissions & NCA/PCA not prescribed or incorrect \\
\hline Incorrect drug levels & Medication prescribed as non-formulary in error \\
\hline Missing/incorrect indication for antibiotics & Regular medication prescribed as a range \\
\hline Other & $\begin{array}{l}\text { Recommended International Non-proprietary } \\
\text { Name (rINN) not specified }\end{array}$ \\
\hline & Other \\
\hline
\end{tabular}

Table 1. Definition of errors 